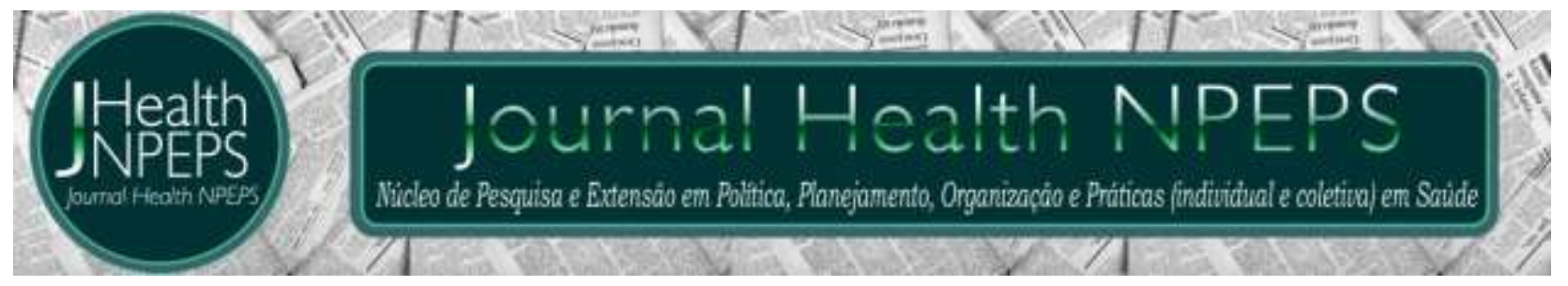

http:/ /dx.doi.org/10.30681/252610102903

ARTIGO ORIGINAL

\title{
Cultura de saúde e segurança dos trabalhadores de um hospital privado
}

\author{
Culture of health and safety of employees of a private hospital
}

\section{Cultura de salud y seguridad de los trabajadores de un hospital privado}

\author{
Renata Alessandra Evangelista ${ }^{1}$, Débora Prado Vasconcelos ${ }^{2}$, Alexandre de Assis \\ Bueno $^{3}$, Luiz Almeida da Silva ${ }^{4}$
}

\begin{abstract}
RESUMO
Objetivo: identificar a percepção dos trabalhadores de um hospital acerca da cultura de saúde e segurança no trabalho. Método: estudo observacional, descritivo, transversal, quantitativo, realizado em um hospital de médio porte, no interior de Goiás. 0 instrumento de coleta foi o Inventário de Clima Organizacional de Segurança. A coleta ocorreu no primeiro semestre de 2016. A análise foi feita através do programa SPSS 20.0. Resultados: participaram do estudo 118 colaboradores, com média de vínculo com a instituição 2,93 anos. Predominantemente do gênero feminino e com idade média de 35 anos. Foi identificado média geral de 2,92 horas de capacitação. Com relação as dimensões do inventário, a equipe de enfermagem apresentou um escore satisfatório nos itens clima de segurança, segurança como valor organizacional e envolvimento pessoal com segurança, enquanto que, nessas mesmas dimensões, a equipe de apoio se caracterizou como indiferente. Na dimensão práticas organizacionais a equipe de enfermagem, mostrou-se indiferente enquanto que a equipe de apoio se apresentou insatisfeita Conclusão: evidenciou-se diferença no envolvimento da percepção da cultura de saúde e segurança no trabalho nesse hospital, ficando evidente a necessidade de capacitação adequada para as categorias que compõem o quadro de trabalhadores. Descritores: Risco Ocupacional; Saúde do Trabalhador; Hospital; Gestão da Segurança.
\end{abstract}

\section{ABSTRACT}

Objetive: to identify the perception of employees of a hospital about the culture of health and safety at work. Method: observational, descriptive, cross-sectional, quantitative study carried out in a medium-size hospital in the interior of Goiás. The instrument of collection was the Organizational Safety Climate Inventory. The

\footnotetext{
${ }^{1}$ Enfermeira. Doutora. Docente no Curso de Enfermagem e Programa de Pós-Graduação em Gestão Organizacional da Universidade Federal de Catalão (UFCat). Catalão-Goiás-Brasil. E-mail: evangelrae@gmail.com ORCID ID: http://orcid.org/0000-0002-2340-1240. Autor principal - Endereço para correspondência: Av. Dr. Lamartine Pinto de Avelar, 1120. Catalão/GO. Brasil

${ }^{2}$ Biomédica. Mestre. Universidade Federal de Catalão (UFCat). Catalão-Goiás-Brasil. E-mail: deborap.vasconcelos@gmail.com ORCID ID: 0000-0002-0964-1459.

${ }^{3}$ Enfermeiro. Mestre. Doutorando do Programa de Enfermagem Fundamental da EERP/USP. Docente Assistente da Universidade Federal de Catalão (UFCat). Catalão-Goiás-Brasil. E-mail: alexissbueno@usp.br ORCID ID: http: //orcid.org/0000-0002-3311-0383.

${ }^{4}$ Enfermeiro. Pós Doutor em Ciências da Saúde. Docente no Curso de Enfermagem e Programa de Pós-Graduação em Gestão Organizacional da Universidade Federal de Catalão (UFCat). Catalão-Goiás-Brasil. E-mail: enferluiz@yahoo.com.br ORCID ID: http://orcid.org/0000-0002-6661-035X.
} 
collection occurred in the first half of 2016. The analysis was done through the SPSS program 20.0. Results: 118 employees participated in the study, with an average of 2.93 years. Predominantly of the feminine gender and with average age of 35 years. A general average of 2.92 hours of training was identified. Regarding the dimensions of the instrument, the nursing team presented a satisfactory score in the items Safety Climate, Safety as organizational value and Personal Involvement with Safety, while, in those same dimensions, the support team was characterized as indifferent. In the Organizational Practices dimension, the Nursing Team was indifferent while the Support Team was dissatisfied. Conclusion: there was a difference the involvement perception of health and safety culture at work. Therefore, the need for adequate and uniform training for the categories that make up the workforce is evident.

Descriptors: Occupational Risk; Worker's health; Hospital; Security Management.

\section{RESUMEN}

Objetivo: identificar la percepción de los trabajadores de un hospital acerca de la cultura de salud y seguridad en el trabajo. Método: estudio descriptivo, transverso, cuantitativo, realizado en un hospital de mediano porte. El instrumento de recolección fue el Inventario de Clima Organizacional de Seguridad. El análisis fue realizado a través del programa SPSS 20.0. Resultados: participaron del estudio 118 colaboradores, con promedio de vínculo con la institución 2,93 años. Predominantemente del género femenino y con edad media de 35 años. Se identificó un promedio general de 2,92 horas de capacitación. Con respecto a las dimensiones del instrumento, el equipo de enfermería presentó una puntuación satisfactoria en los ítems Clima de Seguridad, Seguridad como valor organizacional y Participación Personal con Seguridad, mientras que, en esas mismas dimensiones, el equipo de apoyo se caracterizó como indiferente. En la dimensión Prácticas Organizacionales el Equipo de Enfermería se mostró indiferente mientras que el Equipo de Apoyo se presentó insatisfecho. Conclusión: se evidenció diferencia em la participación de la percepción de la cultura de salud y seguridad en el trabajo en esse hospital, quedando evidente la necesidad de capacitación adecuada para las categorías que componen el cuadro de trabajadores.

Descriptores: Riesgo Ocupacional; Salud del Trabajador; Hospital; Gestión de la Seguridad.

\section{INTRODUÇÃO}

O trabalho, é uma atividade social e exerce papel fundamental nas condições de vida do homem ${ }^{1}$. Por outro lado, ao obtê-lo, o homem se expõe constantemente aos riscos presentes no ambiente de trabalho, os quais podem intervir diretamente em sua condição de saúde².

De todos os trabalhadores, os profissionais que atuam em ambientes hospitalares apresentam maior exposição aos materiais químicos, físicos, biológicos, mecânicos ou de acidentes, psicossociais e ergonômicos, em função da sua rotina profissional ${ }^{3}$.

Atitudes decorrentes desses riscos no ambiente laboral demandam adoção de medidas diversificadas para reduzir o risco ocupacional. Estas são chamadas de 
precauções padrão (PP), dentre elas, o Equipamento de Proteção Individual (EPI) é instrumento essencial para a prevenção de acidentes, no entanto, a aversão do profissional em usá-lo e o seu uso incorreto são grandes obstáculos ${ }^{3}$.

A concepção de uma cultura de saúde e segurança no trabalho, deve permear além das atitudes individuais, um comportamento coletivo, para que a segurança do grupo, pensado como toda a organização, seja refletida e colocada entre as possibilidades de destaque, tanto nos modos de proteção coletiva, como nas individuais. Há que se considerar que a cultura de adesão à segurança tem sido um importante desafio ${ }^{4}$.

Considerando que, a gravidade e frequência dos acidentes, pode ser agravado pela precária infraestrutura, aspectos organizacionais do trabalho, falta de conhecimento devido à não existência de educação continuada, sobrecarga de trabalho, estresse, cansaço físico e ritmo exaustivo de trabalho ${ }^{4}$, tornar-se fundamental identificar a cultura de saúde e segurança entre os trabalhadores, de modo que favoreça o gerenciamento, tomada de decisão e consequentemente a qualidade do serviço prestado.

Sabe-se que o conceito de cultura de segurança pode ser compreendido como um fator decisivo na prevenção de acidentes de trabalho ${ }^{5}$. Contudo, o estudo de clima de segurança, aferindo a percepção dos valores organizacionais, pode significar uma avaliação importante e eficaz no ambiente institucional ${ }^{6}$. Dessa forma, o objetivo do presente estudo foi identificar a percepção dos trabalhadores quanto a cultura de saúde e segurança no trabalho.

\section{MÉTODO}

O estudo caracteriza-se como observacional, quantitativo, descritivo e transversal. Foi realizado em um hospital privado de médio porte, na região da estrada de ferro, no interior de Goiás (GO).

O hospital tem 56 leitos ativos, possui serviços de hemodiálise, hemodinâmica, unidade de terapia intensiva, além de ambulatório de clínica médica e pronto socorro. Atualmente, conta com 223 colaboradores, nas diferentes áreas de atividade.

Para definir os procedimentos e inferir os resultados para a população utilizou-se da técnica de amostragem probabilística aleatória simples, pois cada indivíduo da 
amostra segundo os critérios de inclusão e exclusão teve a mesma probabilidade de ser selecionado. Portanto, foram eleitos todos os trabalhadores que estavam presente na hora que a pesquisa foi realizada e que consentiram em participar assinando o Termo de Consentimento Livre e Esclarecido (TCLE). Foram excluídos os colaboradores ausentes por motivo de férias, licenças ou outras formas de afastamento do serviço no período da coleta dos dados. Após serem submetidos aos critérios de seleção os participantes do estudo totalizaram 118 trabalhadores.

A coleta de dados, ocorreu entre os meses de fevereiro e junho de 2016, utilizando a técnica da entrevista semiestruturada por meio de dois instrumentos, sendo um questionário sobre o perfil sociodemográfico com as seguintes variáveis: idade, gênero, estado civil, escolaridade, turno e tempo de trabalho na instituição e outro referente a percepção da cultura de saúde e segurança, denominado de Inventário de Clima Organizacional de Segurança (ICOS), traduzido e validado7.

O ICOS avalia quatro dimensões: clima de segurança; segurança como valor organizacional; práticas organizacionais de segurança e envolvimento pessoal com segurança. Para tanto, ele utiliza de escalas de Likert de 6 pontos (1 - nada importante até 6 - muitíssimo importante) e de 7 pontos (1 - concordo totalmente até 7 - discordo totalmente).

Este instrumento considera que quanto maior for a pontuação alcançada maior incorporação dos conceitos de saúde e segurança. Foi considerado, que cada alternativa selecionada correspondesse a mesma pontuação, isto é, a opção 1 valeria pontuação 1 , a 2 corresponderia a 2 pontos e assim sucessivamente ${ }^{8}$. Porém, para as perguntas 15, 18, 19, 28, 30, 31, 41, 42, 43 e 44, na escala de 1 a 7, o colaborador que absorvesse conceitos de segurança, escolheria opção 1 como correta, o resultado considerado como positivo seria para maior pontuação, onde a opção 1 corresponderia a 7 pontos, a 2 corresponderia a 6 pontos e assim por diante até a resposta 7 que corresponderia 1 ponto ${ }^{8}$. Contudo, para a análise dos dados, considerou-se de baixa incorporação ou dependendo da análise, insatisfação dos colaboradores escores abaixo de 5 pontos.

O estudo obteve a aprovação do Comitê de Ética em Pesquisa em Seres Humanos, da Universidade Federal de Goiás, conforme Resolução 466/2012, e obteve a aprovação através do Parecer no: 1.362 .720 e aqueles que aceitaram, assinaram TCLE.

Os dados foram tabulados em planilha eletrônica do programa eletrônica do programa estatístico Statistical Package Social Sciences (SPSS), versão 20.0, submetidos 
ao teste de normalidade Kolmogorov-Smirnov e foi feita análise descritiva dos dados com apresentação de médias e desvio-padrão para as variáveis quantitativas e frequência reletiva para as variáveis qualitativas.

Para a análise dos dados, realizou-se primeiramente um teste de confiabilidade do instrumento, no qual utilizou-se o Alpha de Cronbach, e o valor de referência para boa confiabilidade da escala ${ }^{8}$ foi de 0,88 .

\section{RESULTADOS E DISCUSSÃO}

Dentre os participantes, $82,5 \%$ eram do sexo feminino, com idade média de 35,11 anos, casadas $(48,3 \%)$ e trabalham no turno diurno $(61,9 \%)$. Em relação ao tempo de trabalho na instituição, a maioria tinha até 2 anos de serviço. Foi identificado que no ano de 2015, a equipe de enfermagem obteve 3,34 horas de treinamento/capacitação, enquanto que os demais colaboradores obtiveram, 2,5 horas. Particularmente, 60,2\% declaram nunca ter sofrido um acidente de trabalho, conforme Tabela 1.

Tabela 1 - Características sociodemográficas dos colaboradores do hospital. Brasil, 2017. (n=118)

\begin{tabular}{|c|c|c|}
\hline \multirow{2}{*}{\multicolumn{3}{|c|}{ Categoria Funcional* ${ }^{*}$}} \\
\hline & & \\
\hline \multicolumn{3}{|l|}{ Enfermagem } \\
\hline Enfermeiros & 10 & 8,48 \\
\hline Técnicos/Auxiliares & 42 & 35,62 \\
\hline Apoio & 56 & 47,4 \\
\hline Idade (anos) (Média; Mediana) & \multicolumn{2}{|c|}{35,$11 ; 31,5$} \\
\hline \multicolumn{3}{|l|}{ Gênero* } \\
\hline Masculino & 15 & 12,7 \\
\hline Feminino & 100 & 84,7 \\
\hline \multicolumn{3}{|l|}{ Estado civil* } \\
\hline Com companheiro & 57 & 48,3 \\
\hline Sem companheiro & 56 & 47,4 \\
\hline \multicolumn{3}{|l|}{ Escolaridade* } \\
\hline Ensino Médio/Técnico & 70 & 59,3 \\
\hline Graduação & 31 & 26,3 \\
\hline Pós-Graduacão & 10 & 8,5 \\
\hline Tempo de trabalho na instituição* (Média; Mediana) & \multicolumn{2}{|c|}{ 2.97: 2} \\
\hline Até 2 anos & 40 & 33,9 \\
\hline De 2,1 a 4 anos & 21 & 17,8 \\
\hline De 4,1 a 6 anos & 15 & 12,7 \\
\hline De 6,1 a 8 anos & 7 & 5,9 \\
\hline De 8,1 a 10 anos & 3 & 2,5 \\
\hline De 10,1 a 15 anos & 5 & 4,2 \\
\hline Mais de 15 anos & 20 & 16,9 \\
\hline \multicolumn{3}{|l|}{ Turno de trabalho* } \\
\hline Diurno & 73 & 61,9 \\
\hline Noturno & 40 & 33,9 \\
\hline \multicolumn{3}{|l|}{ Cargo de coordenação/chefia* } \\
\hline Sim & 20 & 16,9 \\
\hline
\end{tabular}




\begin{tabular}{lcc}
\hline Não & 93 & 78,8 \\
Treinamento (horas) (Média; Moda; Mediana) & 2,$78 ; 0 ; 0$ \\
Enfermagem (horas) (Média; Moda; Mediana) & 3,$34 ; 0 ; 0$ \\
Apoio (horas) (Média; Moda; Mediana) & 2,$5 ; 0 ; 0$ \\
Acidente de trabalho* & 43 & 36,4 \\
Sim & 71 & 60,2 \\
Não &
\end{tabular}

Fonte: Dados da pesquisa. *Missing

Os profissionais foram divididos em 2 categorias funcionais: Serviços de Enfermagem, que consiste nos profissionais dos setores de Enfermaria, Centro Cirúrgico, Berçário, Hemodiálise, Pronto - Socorro, Unidade de Terapia Intensiva (UTI) e Centro de Diagnóstico de Imagem (CDI); e Apoio, o qual contém os trabalhadores dos setores de Administração, Almoxarifado, Faturamento, Recepção, Farmácia, Serviços de Nutrição e Dietética (Cozinha), Laboratório de Exames, Lavanderia, Serviços Gerais e Internação.

Com relação ao gênero feminino em hospitais, o domínio de mulheres é realidade no contexto hospitalar e, apesar do ingresso dos homens no serviço de enfermagem, a predominância de mulheres permanece 9 . Nesse sentido, em relação a categoria funcional de enfermagem, essa porcentagem de mulheres tem uma relação histórica, pois até o final da Idade Média o papel de cuidadoras era desempenhado por mulheres ${ }^{10}$.

Para a outra categoria profissional, também constatou um alto índice de mulheres $(87,5 \%)$, corroborando com a pesquisa de Santana ${ }^{11}$, que caracterizou os trabalhadores de saúde, incluindo aqueles do serviços de apoio e infraestrutura para a assistência, como os de limpeza, manutenção, apoio diagnóstico e outros, em um hospital de ensino de Curitiba/RS, e também constatou-se a prevalência do gênero feminino, talvez pelo fato de muitas mulheres estar inseridas no mercado de trabalho para contribuir com a renda familiar.

O tempo de trabalho na instituição apresentado foi de 2,93 anos. 0 estudo de Alves $^{12}$, demonstra que cada colaborador representou um referencial sobre a cultura de saúde e segurança do hospital e o tempo de trabalho na instituição um critério dessa representatividade, sendo que um período suficiente para assimilar a cultura de segurança do local de trabalho seria superior a dois anos.

No presente estudo, verificou-se que os colaboradores analisados atingiram essa média, corroborando com o estudo anterior ${ }^{12}$. Dessa maneira, encontrou-se uma 
coerência entre a equipe de enfermagem, a qual apresentou percepção satisfatória em relação aos valores, normas e comportamentos referente ao clima de segurança. No entanto, para a equipe de apoio, mesmo apresentando um tempo de instituição maior que dois anos, não apresentou coesão entre o dado apresentado pela pesquisa de Alves ${ }^{12}$ e os resultados dessa pesquisa (Tabela 2).

Vale ressaltar, o item treinamento/capacitação, que no ano de 2015, no hospital estudado apresentou média geral nas duas categorias funcionais de 2,92 horas por colaborador. Mesmo que se considere apenas a enfermagem, que obteve um valor pouco acima da média geral, essa carga horária de treinamento será equivalente a, aproximadamente, $20 \%$ das 15 horas de treinamento por ano, por funcionário, identificado como ideal, no contexto nacional ${ }^{13}$.

Dos questionários aplicados para a equipe de enfermagem, 52,6\% já sofreram acidente de trabalho e 47,4\% não tiveram treinamento de segurança, sendo que desses $55,56 \%$ são do período diurno e $44,44 \%$ do noturno. Para a equipe de apoio, 21,4\% sofreram acidente de trabalho e $42,9 \%$ não tiveram treinamento de segurança. Esses dados são similares com pesquisa que levantou dados de acidentes de trabalho com os colaboradores do centro de tratamento intensivo adulto (CTI) e lavanderia de um hospital universitário ${ }^{14}$. Os autores desta pesquisa encontraram que os trabalhadores da lavanderia e CTI relataram 19,6\% e 26,5\% respectivamente. Ao questionar sobre o treinamento e prevenção de acidentes, 34,7\% da equipe do CTI relataram que tiveram capacitação/treinamento enquanto que, maior porcentagem de treinamento sobre prevenção de acidentes foi da equipe da lavanderia, apresentando um valor de 98,2\%.

Para a análise das dimensões relativas ao instrumento ICOS, calculou-se a média para cada item com o desvio-padrão, o intervalo de confiança, a variância e a confiabilidade a partir do alfa de Cronbach (Tabela 2).

$\mathrm{Na}$ dimensão Clima de Segurança, verificou-se a capacidade do colaborador em adquirir conhecimento, associado também a percepção sobre a segurança no trabalho ${ }^{15}$, obteve-se média nesse estudo pelos profissionais de enfermagem de 5,12 ( \pm 1$)$, e pela equipe de apoio de 4,19 $( \pm 1,63)$, discordando com um estudo ${ }^{16}$ que avaliou a percepção dos profissionais de saúde sobre a cultura de segurança no centro cirúrgico de um hospital público e obtiveram uma média de 3,42 pontos quanto ao clima de segurança.

E diante de literatura reduzida voltada para colaboradores de hospitais que utilizaram o ICOS, fez-se comparação com estudo sobre a relação do clima 
organizacional de segurança e comportamentos de segurança entre bombeiros do município de Santarém/PA, que apresentou um escore positivo, inclinado à direita do ponto central da escala (escore 4) ${ }^{17}$, corroborando com esta pesquisa. Uma outra pesquisa que verificou a percepção de dentistas a respeito do clima de segurança no trabalho em unidades de atenção básica de saúde no Paraná, também concorda com esta pesquisa, apresentando um escore total de 4,8 pontos ${ }^{6}$.

Essas médias representam o quanto foi incorporado os conceitos sobre valores e normas determinados pela gerência, sendo que os profissionais de enfermagem, tiveram uma maior absorção. Pesquisa referente à saúde e segurança dos trabalhadores em hospitais, no contexto nacional, sugere que escores acima de 5,6 pontos indicam forte consenso entre os colaboradores a respeito do clima de segurança. Todavia, aqueles abaixo de 4,2 significam um sinal de alerta para as instituições, pois indicam a necessidade de ações para implementação de uma cultura de segurança ${ }^{15}$, segundo escala de 1 a 7 pontos.

Tabela 2 - Médias, intervalo de confiança de 95\%, desvio-padrão e variância das dimensões do ICOS da amostra coletada dos colaboradores do hospital. Brasil. 2017. $(n=118)$

\begin{tabular}{|c|c|c|c|c|c|c|c|c|}
\hline \multirow[t]{2}{*}{ Dimensões } & \multicolumn{2}{|c|}{ Média ( \pm DP) } & \multicolumn{2}{|c|}{$\mathrm{IC}^{*}(95 \%)$} & \multicolumn{2}{|c|}{ Variância } & \multicolumn{2}{|c|}{$\begin{array}{l}\text { Alfa- } \\
\text { Cronbach }\end{array}$} \\
\hline & Enf & Apoio & Enf & Apoio & Enf & Apoio & Enf & Apoio \\
\hline $\begin{array}{llll}\begin{array}{l}\text { Conteúdo } \\
\text { segurança }\end{array} & \text { do } & \text { clima } & \text { de } \\
\end{array}$ & $\begin{array}{ll}5,12 \quad( \pm \\
1)\end{array}$ & $\begin{array}{l}4,19 \\
1,63)\end{array}( \pm$ & $\begin{array}{l}4,85- \\
5,38\end{array}$ & $\begin{array}{l}3,76- \\
4,63\end{array}$ & 1 & 2,67 & 0,79 & 0,89 \\
\hline $\begin{array}{l}\text { Percepção de valores } \\
\text { determinados pela gerência }\end{array}$ & $\begin{array}{l}4,93 \quad( \pm \\
0,89)\end{array}$ & $\begin{array}{l}3,98 \\
1,6)\end{array} \quad( \pm$ & $\begin{array}{l}4,7- \\
5,17\end{array}$ & $\begin{array}{l}3,55- \\
4,41\end{array}$ & 0,8 & 2,57 & & \\
\hline $\begin{array}{l}\text { Percepção de normas sobre os } \\
\text { comportamentos esperados }\end{array}$ & $\begin{array}{l}5,3 \\
1,28)\end{array}$ & $\begin{array}{l}4,41 \\
1,83)\end{array}$ & $\begin{array}{l}4,96- \\
5,64\end{array}$ & $\begin{array}{l}3,92- \\
4,9\end{array}$ & 1,64 & 3,35 & & \\
\hline $\begin{array}{l}\text { Segurança como } \\
\text { organizacional }\end{array}$ & $\begin{array}{l}5,09 \\
1,53)\end{array}$ & $\begin{array}{l}4,07 \\
1,88)\end{array}$ & $\begin{array}{l}4,68 \\
5,5\end{array}$ & $\begin{array}{l}3,57 \\
4,57\end{array}$ & 2,34 & 3,53 & 0,92 & 0,92 \\
\hline $\begin{array}{l}\text { Práticas organizacionais de } \\
\text { segurança }\end{array}$ & $\begin{array}{l}4,52 \\
1,04)\end{array}( \pm$ & $\begin{array}{l}3,6 \\
1,34)\end{array}( \pm$ & $\begin{array}{l}4,24- \\
4,79\end{array}$ & $\begin{array}{l}3,38 \\
4,12\end{array}$ & 1,08 & 1,92 & 0,86 & 0,91 \\
\hline $\begin{array}{l}\text { Gerenciamento das atividades de } \\
\text { segurança }\end{array}$ & $\begin{array}{l}4,47 \\
1,45)\end{array}$ & $\begin{array}{l}3,92 \\
1,64)\end{array}$ & $\begin{array}{l}4,09- \\
4,86\end{array}$ & $\begin{array}{l}3,48- \\
4,36\end{array}$ & 2,12 & 2,69 & & \\
\hline Treinamento de segurança & $\begin{array}{l}4,15 \\
1,36)\end{array}$ & $\begin{array}{l}3,55 \\
1,56)\end{array}$ & $\begin{array}{l}3,78 \\
4,51\end{array}$ & $\begin{array}{l}3,13- \\
3,97\end{array}$ & 1,84 & 2,44 & & \\
\hline Efetividade da segurança & $\begin{array}{l}4,67 \\
1,47)\end{array}$ & $\begin{array}{l}3,65 \\
1,66)\end{array}$ & $\begin{array}{l}4,28 \\
5,06\end{array}$ & $\begin{array}{l}3,2- \\
4,09\end{array}$ & 2,17 & 2,76 & & \\
\hline $\begin{array}{l}\text { Qualidade das comunicações de } \\
\text { segurança }\end{array}$ & $\begin{array}{l}4,23 \\
1,08)\end{array}$ & $\begin{array}{l}3,65 \\
1,56)\end{array} \quad( \pm$ & $\begin{array}{l}3,95 \\
4,52\end{array}$ & $\begin{array}{l}3,27- \\
4,03\end{array}$ & 1,16 & 2,01 & & \\
\hline $\begin{array}{l}\text { Efeitos de ritmo de trabalho } \\
\text { solicitado }\end{array}$ & $\begin{array}{l}4,74 \\
1,33)\end{array}$ & $\begin{array}{l}4,12 \\
1,77)\end{array}$ & $\begin{array}{l}4,39 \\
5,09\end{array}$ & $\begin{array}{l}3,65- \\
4,6\end{array}$ & 1,76 & 3,14 & & \\
\hline $\begin{array}{l}\text { Aprendizado organizacional a } \\
\text { partir de acidentes }\end{array}$ & $\begin{array}{l}4,85 \\
1,39)\end{array}$ & $\begin{array}{l}3,61 \\
1,87)\end{array}$ & $\begin{array}{l}4,48 \\
5,22\end{array}$ & $\begin{array}{l}3,11- \\
4,11\end{array}$ & 1,93 & 3,51 & & \\
\hline $\begin{array}{l}\text { Envolvimento pessoal com } \\
\text { segurança }\end{array}$ & $\begin{array}{l}5,18 \\
1,17)\end{array}$ & $\begin{array}{l}4,15 \\
1,71)\end{array}$ & $\begin{array}{l}4,87- \\
5,49\end{array}$ & $\begin{array}{l}3,69- \\
4,61\end{array}$ & 1,38 & 2,94 & 0,72 & 0,85 \\
\hline $\begin{array}{l}\text { Comprometimento pessoal com } \\
\text { segurança }\end{array}$ & $\begin{array}{l}5,09 \\
1,5)\end{array}$ & $\begin{array}{l}3,85 \\
1,97)\end{array}$ & $\begin{array}{l}4,69 \\
5,49\end{array}$ & $\begin{array}{l}3,32 \\
4,38\end{array}$ & 2,26 & 3,87 & & \\
\hline Internalização de segurança & $4,96 \quad( \pm$ & $4,18 \quad( \pm$ & 4,59 & $3,69-$ & 1,87 & 3,37 & & \\
\hline
\end{tabular}




\begin{tabular}{|c|c|c|c|c|c|c|c|c|}
\hline Orgulho quanto a segurança & $\begin{array}{l}1,37) \\
5,49 \\
1,52)\end{array}$ & $\begin{array}{l}1,83) \\
4,43 \\
2,05)\end{array}$ & $\begin{array}{l}5,32 \\
5,09- \\
5,89\end{array}$ & $\begin{array}{l}4,67 \\
3,88- \\
4,98\end{array}$ & 2,32 & 4,21 & & \\
\hline Escore Global & $\begin{array}{l}4,98 \\
0,98)\end{array} \quad( \pm$ & $\begin{array}{l}3,8 \\
1,41)\end{array} \quad( \pm$ & $\begin{array}{l}4,71- \\
5,24\end{array}$ & $\begin{array}{l}3,42- \\
4,17\end{array}$ & 0,97 & 1,99 & 0,83 & 0,89 \\
\hline
\end{tabular}

Fonte: Dados da pesquisa. *Intervalos de Confiança.

Em um estudo ${ }^{16}$, que teve como proposta investigar a percepção do clima de segurança entre os profissionais de enfermagem (enfermeiros, auxiliares e técnicos de enfermagem), observou que a percepção quanto ao clima de segurança variou, de acordo com a categoria profissional. E constatou que enfermeiros apresentam maiores escores se comparado aos técnicos e auxiliares de enfermagem. Contudo, no presente estudo, não foi encontrado diferença significativa entre as percepções de clima de segurança dos enfermeiros com os técnicos e auxiliares de enfermagem.

Com relação aos dados referente a Segurança como valor organizacional, ao analisar os resultados dessa pesquisa, observa-se que a enfermagem assimilou estes conceitos no seu cotidiano, apresentando uma média de 5,09 $( \pm 1,53)$, enquanto o apoio, obteve uma média de 4,07 $( \pm 1,88)$, resultado satisfatório quando comparado ao estudo que avaliou a percepção dos profissionais de saúde sobre a cultura de segurança no centro cirúrgico de um hospital público e obtiveram uma média de 3,74 pontos, conforme escala de 1 a 7 pontos ${ }^{17}$. Para esses autores, é indispensável mensurar a segurança por meio da percepção dos colaboradores e avaliar os resultados para efetivar melhorias no cenário hospitalar.

Outra dimensão avaliada é Práticas organizacionais de segurança que está vinculada a percepção dos respondentes quanto gerenciamento das atividades de segurança; treinamento de segurança; efetividade da segurança; qualidade das comunicações de segurança; efeitos de ritmo de trabalho solicitado; e aprendizado organizacional. No presente estudo, a média apresentada pelos profissionais de enfermagem foi de 4,52 ( \pm 1,04) que representa uma indiferença com as práticas organizacionais acerca da segurança, enquanto que a equipe de apoio obteve o índice de 3,6 $( \pm 1,34)$, revelando insatisfação (Tabela 2).

A partir destes resultados, pode-se inferir que para obter êxito no exercício do gerenciamento da segurança no ambiente laboral, precisa necessariamente, incluir um programa de treinamento e/ou capacitação/educação continuada. No contexto hospitalar, esse gerenciamento refere-se à organização do trabalho, normas, protocolos, 
ritmo de trabalho e qualidade da comunicação, a qual é diretamente ligada ao nível de aprendizado dos colaboradores ${ }^{17}$.

Enquanto o estudo de Carvalho ${ }^{17}$, apresentou um resultado de 2,41 pontos, feito com os trabalhadores do Hospital de Base do Distrito Federal, no estudo de Couto, realizado com bombeiros no distrito de Santarém/PA foi obtido uma média de 4,65 pontos quanto ao gerenciamento ${ }^{18}$. Essa oscilação sugere que este domínio acompanha o modelo de gestão aplicado em cada instituição ${ }^{19}$. Todavia, um baixo escore quanto a percepção em relação ao gerenciamento das atividades de segurança remete a ideia de que os colaboradores que atuam no cenário avaliado não percebem o compromisso da gestão com a cultura de segurança.

Outro item a observar é a qualidade das comunicações de segurança, em que se obteve média para os profissionais de enfermagem de $4,23( \pm 1,08)$ e para a equipe de apoio de 3,65 $( \pm 1,56)$. Estes resultados representam uma insatisfação e baixa incorporação dos colaboradores, e desta forma, as mesmas podem ser úteis para os gestores da área, pois direcionam para a promoção de uma comunicação adequada entre suas equipes.

Uma pesquisa identificou a percepção de enfermeiros sobre a utilização dos indicadores, referente a gestão da qualidade e consequentemente os pontos fortes e fracos neste nível de comunicação entre os trabalhadores ${ }^{20}$. Eles encontraram que a maioria de seus respondentes discordaram que a comunicação no referido hospital é de forma adequada, atribuindo escore baixo neste item. Já em outro estudo, a média encontrada para a qualidade das comunicações de segurança foi de 4,34, sendo esta a média mais baixa dentre os itens da dimensão Práticas Organizacionais de Segurança ${ }^{18}$, corroborando com este estudo.

$\mathrm{Na}$ análise da dimensão Envolvimento pessoal com segurança, a média apresentada foi de 5,18 $( \pm 1,17)$ para os profissionais de enfermagem e de 4,15 $( \pm 1,71)$ para a equipe de apoio, demonstrando uma tendência a indiferença quanto a satisfação dos colaboradores.

Em um estudo sobre as evidências do trabalho em equipe na atenção hospitalar, considerou-se imprescindível o contentamento do colaborador referente a sua importância, qualidade e desempenho, já que fazem parte do processo dinâmico e inseparável no ambiente de trabalho ${ }^{21}$. Eles acreditam que essa dimensão, envolvimento 
pessoal com a segurança, está intimamente ligada ao estado fisiológico e psicológico referente a segurança do colaborador.

Nesta dimensão, enquanto o estudo do Couto $^{18}$ identifica uma média de 5,7 pontos entre seus colaboradores, outro estudo feito com bancários do município de Araçatuba/SP em quatro agências bancárias, encontrou uma média de 3,31 pontos ${ }^{22}$. Acredita-se que essa baixa adesão é percebida como uma atitude dos colaboradores de restabelecerem sua segurança frente um cenário de mudanças institucionais.

Por fim, ao realizar a análise do Escore global, obteve-se uma média de 4,98 $( \pm$ 0,98) para os profissionais de enfermagem e uma média de 3,8 $( \pm 1,41)$ para a equipe de apoio. Esse valor representa que enquanto a enfermagem absorveu quase $50 \%$ da cultura de saúde e segurança do trabalho, a equipe apoio apresentou 38\% de adesão.

Além disso, constatou-se diferença entre as percepções gerais dos profissionais de enfermagem, sendo que os enfermeiros obtiveram escores maiores em relação aos técnicos e auxiliares de enfermagem, corroborando com o trabalho de Rigobello ${ }^{15}$.

Esse resultado apresenta avaliação moderada da percepção dos colaboradores quanto a sua saúde e segurança no trabalho, confirmando pesquisa que discute a saúde e segurança de enfermeiros de unidade de tratamento intensivo em um hospital privado no Rio de Janeiro, a partir de três fatores que avaliam os riscos de adoecimento no trabalho, sendo eles: 1. organização do trabalho, subdividido em divisão e conteúdo das tarefas, normas, controles e ritmos de trabalho; 2. relações sócio profissionais, subdividida em modos de gestão do trabalho, comunicação e interação profissional; e 3. Condições de trabalho, subdividido em qualidade do ambiente físico, posto de trabalho, equipamentos e material disponibilizados para a execução do trabalho. Eles obtiveram um escore médio total de 2, 75 pontos, em uma escala de 1 a 5 pontos, para a percepção do trabalhador sobre sua saúde e segurança no ambiente de trabalho ${ }^{19}$. A avaliação desse dado é dada pelos pesquisadores como moderada crítica.

Ressalta-se que a equipe de apoio obteve nas dimensões avaliadas do ICOS um comportamento indiferente ou de insatisfação, isto é, a opção da categoria central, do tipo "nem satisfeito, nem insatisfeito", pode revelar indiferença dos respondentes, significando uma distorção da verdadeira opinião ou que eles não têm condições de opinar ${ }^{23}$.

Destaca-se que o estudo apresentou algumas limitações, por ter sido realizado apenas em uma unidade hospitalar e por não terem participado todos os colaboradores. 
Além disso, houve artigos limitados na área hospitalar, os quais utilizaram o mesmo instrumento e que poderiam enriquecer a discussão.

\section{CONCLUSÃO}

A presente pesquisa buscou apresentar a percepção dos colaboradores acerca da saúde e segurança no trabalho. A observação deste construto permite um diagnóstico da cultura de segurança e conscientização dos trabalhadores acerca do tema; avaliação de intervenções para a segurança implementadas na organização e o acompanhamento ao longo dos anos.

Com intuito de contribuir como estratégia de promoção da saúde, e fortalecimento da cultura de segurança, os resultados nos apontaram em que direção a instituição deve organizar sua ações e planejamento.

Os resultados mostraram diferença no envolvimento e percepção da cultura de saúde e segurança no trabalho entre os profissionais de enfermagem e equipe de apoio. Ficou evidente a necessidade de capacitação adequada para as categorias que compõem o quadro de colaboradores.

Com relação a equipe de apoio, nas dimensões clima de segurança e práticas organizacionais de segurança revelaram uma insatisfação, o que nos leva a inferir que há necessidade de aprofundar a compreensão sobre os conjuntos de aspectos que colaboram para isso. Tal dimensão não se consolida sem superar problemas de capacitação/educação continuada, qualidade nas comunicações de segurança, efetividade da segurança e aprendizado organizacional. Portanto, considera-se que os resultados encontrados demandam consideração dos coordenadores/chefia.

\section{REFERÊNCIAS}

1. Bottaro BB, Pereira FMV, Reinato LAF, Canini SRMS, Malaguti-Toffano SE, Gir E. Adesão às precauções-padrão pelos profissionais de enfermagem: uma revisão da literatura. Rev enferm UFPE on line. 2016; 10(3):1137-42.

2. Cavaleiro MR, Ferreira A, Figueiredo J, Conde J. Estudo da sinistralidade laboral em meio hospitalar - Aspecto fundamental para a integração da segurança no trabalho na gestão de unidades de saúde. Int J Work Conditions. 2012; (3):21-41. 
3. Reis FRD, Kitamura S. O controle estatal em saúde e segurança no trabalho e a auditoria do programa de controle medico de saúde ocupacional. Rev Bras Med Trab. 2016; (14):52-9.

4. Camposa DC, Diasa MCF. A cultura de segurança no trabalho: um estudo exploratório. Sist gest. 2012; 7(4):594-604.

5. Silva MC, Borges LO. Condições de trabalho e clima de segurança dos operários da construção de edificações. Rev Psicol Organ Trab. 2015; 15(4):407-18.

6. Ribeiro PHV, Brevidelli MM, Tipple AFV, Ribeiro RP, Gir E. Clima de segurança organizacional e a adesão às precauções padrão entre dentistas. Acta Paul Enferm. 2013; 26(2):192-7.

7. Silva S, Lima ML, Baptista C. OSCl: an organizational and safety climate inventory. Saf sci. 2004; 42(3):205-20.

8. Nickhorn MM, Sellitto MA. Análise Comparativa da Aplicação de Sistemas de Gestão de Segurança em Empresas da Indústria Automotiva. GEINTEC. 2015; 5(4):2703-17.

9. Souza SBC, Tavares JP, Macedo ABT, Moreira PW, Lautert L. Influência do turno de trabalho e cronótipo na qualidade de vida dos trabalhadores de enfermagem. Rev Gaúch Enferm. 2012; 33(4):79-85.

10. Souza LL, Araújo DB, Silva DS, Bêrredo VCM. Representações de gênero na prática de enfermagem na perspectiva de estudantes. Ciênc cogn. 2014;19(2):218-32.

11. Santana LL, Miranda FAD, Karino ME, Baptista PCP, Felli VEA, Sarquis LMM. Cargas e desgastes de trabalho vivenciados entre trabalhadores de saúde em um hospital de ensino. Rev Gaúch Enferm. 2013; 34(1):64-70.

12. Alves SMP, Coelho MCR, Borges LH, Cruz CAM, Massaroni L, Maciel PMA. A flexibilização das relações de trabalho na saúde: a realidade de um Hospital Universitário Federal. Ciênc Saúde Colet. 2015; 20(10):3043-50.

13. Alves DFS, Almeida AO, Higa R, Jorge SA. Indicador de treinamento em educação continuada para enfermagem: análise em um hospital universitário. Rev Eletrônica SIMTEC. 2016; (6):49-53.

14. Silva EJ, Lima MG, Marziale MHP. O conceito de risco e os seus efeitos simbólicos nos acidentes com instrumentos perfuro cortantes. Rev Bras Enferm. 2012; 65(5):809-14.

15. Ribobello MCG, Carvalho REFL, Cassiani SHB, Galon T, Capucho HC, Deus NN. Clima de segurança do paciente: percepção dos profissionais de enfermagem. Acta Paul Enferm. 2012; 25(5):728-35. 
16. Andrade AL, Silva MZ, Netto MT. Clima de Segurança no Trabalho Hospitalar: adaptação de medida (ClimaSeg-H). Aval psicol. 2015; 14(2):261-71.

17. Carvalho PA, Götems LBD, Pires MRGM, Oliveira MLC. Cultura de segurança no centro cirúrgico de um hospital público, na percepção dos profissionais de saúde. Rev LatinoAm de Enf. 2015; 23(6):1041-148.

18. Guirardello EB. Impacto do ambiente de cuidados críticos no burnout, percepção da qualidade do cuidado e atitude de segurança da equipe de enfermagem. Rev Latino-Am Enferm. 2017; 25:e2884.

19. Françolin L, Gabriel CS, Bernardes A, Silva AEBC, Brito MFP, Machado JP, et al. Gerenciamento da segurança do paciente sob a ótica dos enfermeiros. Rev Esc Enferm USP. 2015; 49(2):277-83.

20. Silveira TVL, Prado Júnior PP, Siman AG, Amaro MOF. Opinião dos enfermeiros sobre a utilização dos indicadores de qualidade na assistência de enfermagem. Rev Gaúch Enferm. 2015; 36(2):82-8.

21. Alves LR, Giacomini MA, Camelo SH, Laus AM, Leal LA, Goulart BF, et al. Evidências sobre o trabalho em equipe na atenção hospitalar. J Health NPEPS. 2016; 1(2):246-62.

22. Reatto D, Silva DA, Isidoro ML, Rodrigues NT. Prevalência da Síndrome de Burnout no setor bancário no município de Araçatuba (SP). Arch health invest. 2014; 3(2):1-8.

23. Dalmoro M, Vieira KM. Dilemas Na Construção De Escalas Tipo Likert: O Número De Itens E A Disposição Influenciam Nos Resultados? Rev gest org. 2013; 6(3):161-74.

Conflito de interesses: Os autores declaram não haver conflito de interesses.

Participação dos autores: Os autores declaram que participaram de todas as etapas do estudo (concepção, desenvolvimento do estudo, redação e revisão).

Como citar este artigo: Evangelista RA, Vasconcelos DP, Bueno AA, Silva LA. Cultura de saúde e segurança dos trabalhadores de um hospital privado. Journal Health NPEPS. 2018; 3(1):118-131. 